\title{
Natural History of Patients with Unresectable Cholangiocarcinoma
}

\author{
Sudhakar A $\quad$ Sravan Kumar K ${ }^{1} \quad$ L. R.S. Girinadh ${ }^{1}$ \\ ${ }^{1}$ Department of Gastroenterology, KGH, Visakhapatnam, \\ Andhra Pradesh, India
}

J Digest Endosc 2020;10:206-209

\author{
P. MuraliKrishna ${ }^{1}$
}

\begin{abstract}
Address for correspondence Dr. Sravan Kumar K, MD, Department of Gastroenterology, KGH, Visakhapatnam, Andhra Pradesh 530002, India (e-mail: Sravan8dham.skk@gmail.com).
\end{abstract}

\author{
Abstract \\ Keywords \\ - cholangiocarcinoma \\ - jaundice \\ - survival \\ - biliary drainage
}

Cholangiocarcinoma is a malignancy of the bile ducts. Globally, CCA is the second most common primary hepatic malignancy. These tumors usually progress insidiously, are difficult to diagnose, and have poor prognosis. Unfortunately, treatment options are discouraging. Data on the natural history of CCA in coastal Andhra Pradesh is limited. Our aim is to report the natural history of CCA in this region.

\section{Introduction}

Cholangiocarcinoma (CCA) is a malignancy of the bile ducts. Globally, CCA is the second most common primary hepatic malignancy. ${ }^{1,2}$ From the anatomical point of view, CCA is classified as intrahepatic (IHCCA) or extrahepatic (EHCCA), based on its location along the biliary tree. Extra hepatic CCA is further divided into proximal (perihilar) and distal. The second-order bile ducts serve as the point of separation between IHCCAs and perihilar CCAs (pCCAs). The cystic duct forms the anatomic boundary between perihilar and distal CCAs.

Patients with EHCCA have symptoms, signs, and biochemical laboratory tests of obstructive cholestasis. IHCCA has nonspecific symptoms. An incidental abdominal mass during physical examination or imaging study may be the sole finding in asymptomatic patients. Surgical resection is a potentially curative treatment for both EHCCA and IHCCA. Unfortunately, more than half of CCA patients have advanced unresectable disease. In such cases, palliative therapies (i.e., biliary stenting and photodynamic therapy) provide symptom relief and may have a positive effect on survival rates. The very small fraction of selected patients with CCA may undergo orthotopic liver transplantation (OLT) with curative intent.
Data on the natural history of CCA in coastal Andhra Pradesh is limited. Our aim is to report the natural history of CCA in this region.

\section{Materials and Methods}

Long-term follow-up of patients with CCA undergoing endoscopic therapy was performed by retrospective analysis. The study was conducted from January 2013 to December 2016 in the Department of Gastroenterology at King George Hospital, Visakhapatnam. Data acquisition was based on hospital records. Furthermore, follow-up data was obtained by telephone contact with relatives of the patients. To evaluate the life expectancy of patients with CCA, follow-up analysis was carried out from the time when the patient received his or her first treatment until death. Baseline characteristics were age, gender, bilirubin levels, alkaline phosphatase, leucocyte count, and type of therapeutic procedures including endoscopic transpapillary drainage and percutaneous transhepatic drainage.

All the patients underwent either computer tomography (CT) scan, magnetic resonance imaging (MRI), magnetic resonance cholangiopancreatography (MRCP), or endoscopic retrograde cholangiography (ERCP) for diagnosis and staging of the disease. Serum tumor markers such as CA 19-9 
and CEA were measured in selected patients. The final diagnosis was made by surgical specimens, percutaneous ultrasound-guided fine needle biopsy, endoscopic transpapillary forceps biopsy/brush cytology, and percutaneous transhepatic cholangioscopic-guided biopsy. In the absence of a tissue diagnosis, a clinical diagnosis was established based on clinical symptoms, levels of tumor markers, imaging studies$\mathrm{CT}$, MRI, MRCP including ERCP, and particularly the course of the disease. Staging of the disease was done according to the TNM (tumor, node, and metastasis) classification.

The type of therapeutic procedures depended on tumor stage and clinical condition of patients. If the tumor was resectable, surgery was the first choice of treatment for patients with good performance status. In all patients, the possibility of a curative therapy concept with tumor resection was evaluated. In patients with nonresectable tumors or poor performance status, palliative drainage was performed. Systemic chemotherapy was administered in advanced disease wherever feasible.

ERCP was done by a standard videoduodenoscope Olympus TJF 150-R (Olympus, Japan). After guidewire cannulation, under radiographic guidance using contrast fluid, bile duct strictures were localized. Subsequently, one or more plastic endoprostheses were placed above the stricture to obtain biliary drainage. The caliber of stents varied between $7 \mathrm{~F}$ and $10 \mathrm{~F}$. In patients in whom palliative biliary drainage is needed, wherever feasible, metal stenting (covered/uncovered) was done for adequate drainage. Covered stents were used where the stricture was below the level of cystic duct. Uncovered stents were used when the stricture was at or near the hilum. The size of the stent was decided based on the distance from papilla at the time of ERCP. Elective stent changes were conducted at a time interval of 3 months unless the clinical situation of patients required an earlier intervention.

\section{Statistical Analysis}

Survival in relation to bilirubin, TNM stage, and type of endoscopic procedures (ERC versus PTBD) were estimated by the Kaplan-Meier curves. Statistical significance was considered at $p<0.05$.

\section{Results}

A total of 86 CCA patients underwent treatment. Sixteen patients underwent surgery, who were excluded from the analysis. Among the 70 patients, 34 were men and 36 were female. The mean age was 53.43 years. The clinical presentation in these patients was jaundice (85.7\%), abdominal pain (21.4\%), pruritus (60\%), and anorexia and weight loss (35.7\%) (-Fig. 1). Six patients presented with cholangitis (8.5\%). Eight patients $(11.4 \%)$ were found to be having an intrahepatic mass on imaging done for the evaluation of anorexia and abdominal pain. Two patients had biliary stricture without jaundice found on imaging done for evaluation of pain abdomen. The most common risk factors identified were smoking followed by alcohol, hepatitis C virus (HCV) infection, cirrhosis, and gallstones ( - Fig. 2). None of the patients had a family history of malignancy.

Nine patients had an elevated total leucocyte count of greater than $10,000 / \mathrm{m}^{3}$. Six of these nine patients had symptoms of cholangitis at presentation. The average serum bilirubin levels were $9.71 \mathrm{mg} / \mathrm{dL}$ and the average alkaline phosphatase levels were $298 \mathrm{U} / \mathrm{dL}$. Fourteen patients had

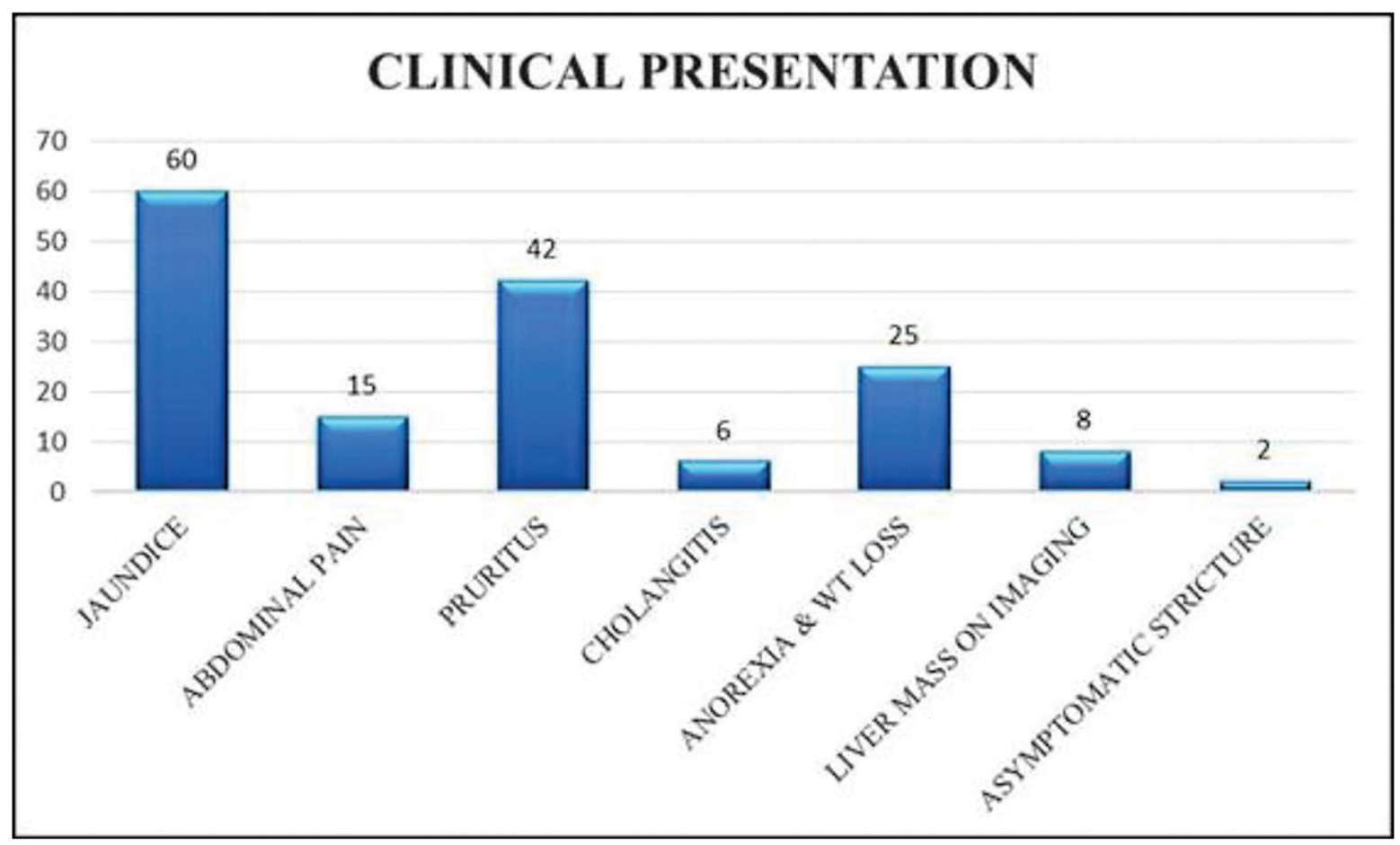

Fig. 1 Clinical presentation in cholangiocarcinoma. 


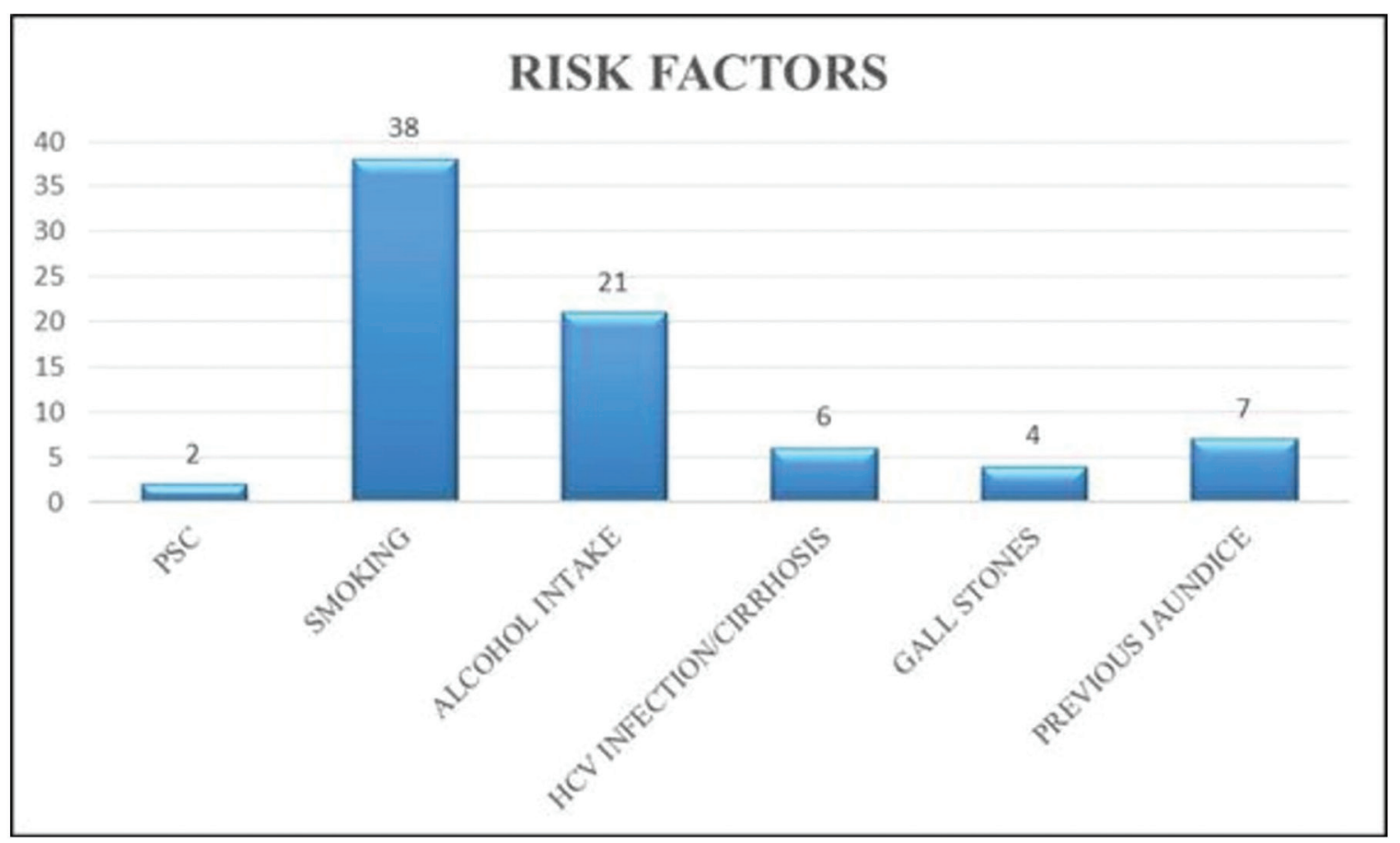

Fig. 2 Risk factors in cholangiocarcinoma.

elevated aminotransferase levels at presentation. Based on tumor location, eight patients had intrahepatic CCA, 41 patients had perihilar CCA, and 21 patients had distal CCA. Patients with perihilar CCA were further classified according to Bismuth-Corlette classification. Sixteen patients were afflicted with Type I pCCA, 11 patients had Type II pCCA, three patients had Type IIIa pCCA, two patients had Type IIIb pCCA, and one patient had Type IV pCCA. Among eight patients, perihilar CCA was not classified.

Fifty-two patients underwent palliative endoscopic biliary drainage and 18 patients underwent percutaneous transhepatic drainage. None received chemoradiation. The overall mean survival of patients was 6.7 months. Fifty-eight patients out of a total of 70 died during the follow-up period with a one-year mortality rate of $82.85 \%$.

The mean survival of patients with an initial bilirubin of more than $10 \mathrm{mg} / \mathrm{dL}(n=49)$ was $5.8 \pm 3.1$ months. The mean survival of patients with an initial bilirubin of less than $10 \mathrm{mg} / \mathrm{dL}(n=21)$ was $7.9 \pm 3.9$ months. The mean survival time of patients with initial bilirubin levels more than $10 \mathrm{mg} / \mathrm{dL}(5.8 \pm 3.1$ months $)$ was significantly lower $(p<0.01)$ than patients with bilirubin levels less than $10 \mathrm{mg} / \mathrm{dL}$ (7.9 \pm 3.9 months).

The mean survival time of patients with TNM stage II ( $n=18)$, III $(n=22)$, and IV ( $n=30)$ was $6.6 \pm 4.4$ months, $6.0 \pm 3.4$ months, and $5.2 \pm 3.2$ months, respectively. Thus, patients with advanced TNM stage showed a reduced mean survival time. The mean survival of patients with TNM stage II was not significantly higher than stage III $(p=0.6)$ and stage IV $(p=0.8)$. The mean survival of patients was not significantly different between stage III and stage IV $(p=0.2)$.
There was no significant difference in survival between patients who underwent transpapillary endoscopic drainage and percutaneous drainage $(p=0.8)$.

\section{Discussion}

CCA is the second most common primary hepatic cancer. Although its overall incidence is low, it is on the rise globally. ${ }^{2} \mathrm{CCA}$ has a dismal prognosis, with a 5 -year survival rate of less than $10 \%$.

Risk factors for the development of CCA are primary sclerosing cholangitis (PSC), chronic choledocholithiasis, liver cirrhosis, bile duct adenoma, and other rare diseases such as biliary papillomatosis, Caroli's disease, choledochal cyst, and parasitic biliary infestation. ${ }^{3-5}$

Perihilar CCA accounted for $58.6 \%$ of overall cases of CCA and $66.1 \%$ of extrahepatic CCA cases. Distal CCA accounted for $30 \%$ of CCA cases and $33.9 \%$ of extrahepatic CCA cases.

In this study, bilirubin level at initial presentation was a significant prognostic parameter. Our results are similar to the study carried out by Prinz et al. ${ }^{6}$

Surgical resection is a mainstay of treatment with curative intent. However, previous reports indicate that not all patients are able to undergo a surgical treatment, and thus palliative options can be offered in a lot of cases. ${ }^{78}$ Palliative treatment strategies including ERCP, routinely performed in our hospital, experienced a few complications only, and were safe and effective measures to improve excretion of bile fluid. There was no significant difference in the effectiveness between ERCP and percutaneous transhepatic cholangiography (PTC). We have not evaluated other palliative therapies like photodynamic therapy. 
To our knowledge, this is the first study in our country, evaluating the natural history and prognostic factors of CCA.

In conclusion, CCA has a similar incidence in both sexes with a mean age of presentation in the 6th decade. Jaundice and weight loss is the most common presentation. Smoking and alcoholism are significant risk factors identified along with PSC. Initial bilirubin level is a significant prognostic factor for survival. In contrast, tumor stage according to the TNM and type of drainage procedure are not significant prognostic parameters for survival.

\section{Financial support}

No.

\section{Informed Consent of the Patient}

Obtained.

\section{Conflict of Interest}

None declared.

\section{References}

1 Shaib Y, El-Serag HB. The epidemiology of cholangiocarcinoma. Semin Liver Dis 2004;24(2):115-125

2 Patel T. Increasing incidence and mortality of primary intrahepatic cholangiocarcinoma in the United States. Hepatology 2001;33(6):1353-1357
3 Shaib YH, El-Serag HB, Davila JA, Morgan R, McGlynn KA. Risk factors of intrahepatic cholangiocarcinoma in the United States: a case-control study. Gastroenterology 2005;128(3): 620-626

4 Gores GJ. A spotlight on cholangiocarcinoma. Gastroenterology 2003;125(5):1536-1538

5 Gores GJ. Cholangiocarcinoma: current concepts and insights. Hepatology 2003;37(5):961-969

6 Weber A, Landrock S, Schneider J, et al. Long-term outcome and prognostic factors of patients with hilar cholangiocarcinoma. World J Gastroenterol 2007;13(9):1422-1426

7 Lang H, Sotiropoulos GC, Frühauf NR, et al. Extended hepatectomy for intrahepatic cholangiocellular carcinoma (ICC): when is it worthwhile? Single center experience with 27 resections in 50 patients over a 5-year period. Ann Surg 2005;241(1): 134-143

8 Witzigmann $\mathrm{H}$, Berr F, Ringel U, et al. Surgical and palliative management and outcome in 184 patients with hilar cholangiocarcinoma: palliative photodynamic therapy plus stenting is comparable to r1/r2 resection. Ann Surg 2006;244(2):230-239 